\title{
Validación del instrumento del Marketing Relacional "RM" en los clientes de la empresa Cinemark, Surco,2020
}

Validation of the "RM" Relationship Marketing instrument in the clients of the Cinemark company, Surco, 2020

Miguel Angel Gallegos Reyes ${ }^{a *}$, Jose Alberto Panduro Ramirez ${ }^{b}$, Alexander David de la Cruz Vargas

a,b,c Universidad Peruana Unión, Lima, Perú

\section{Resumen}

El objetivo de la presente investigación es determinar la consistencia interna y confiabilidad del instrumento Relationship Marketing, mediante un análisis exploratorio. El instrumento creado por Ndubisi (2007) calcula las dimensiones, confianza, compromiso, comunicación y manejo de conflictos mediante 16 reactivos. Dentro de los análisis previos de consistencia interna se obtuvo un Alfa de Cronbach para el instrumento corresponde $\square=0.883$ y 0.458 , $0.789,0.657,0.821$ para sus dimensiones. De esta forma para constatar la homogeneidad de las variables (reactivos), con anticipación se realizaron las estimaciones mediante $\mathrm{KMO}$, alcanzando un índice de Káiser Meyer Olkin admisible de 0.816 y un Chi cuadrado de 695.946 con 120 grados de libertad y nivel de significancia $=0.000(p<.001)$ mediante la prueba de esfericidad de Bartlett, como requisito indispensable para la extracción de factores mediante componente rotados, adquiriendo para el primer elemento cargas entre 0.780 y 0.790 ; para el segundo elemento, entre 0.738 y 0.779 , para el tercer elemento, entre 0.739 y 0.652 y para el cuarto elemento 0.598, justificando una alta correlación, porque son mayores a 0.4 , formalizando el objetivo de revisar si se agrupan empíricamente, tal como, teóricamente se había pronosticado. Sin embargo, el reactivo COMUN 10 (0.486), COMUN 11, (0.482), CONFL15 (0.148) no obtuvieron carga en su dimensionalidad y los reactivos CONFI 5 (0.385), COMUN13 (0.244), CONFL16 (0.498) obtuvieron cargas adicionales en otros elementos, pudiendo ser eliminados, se decidió mantenerlos en su factor teórico, esperando un análisis confirmatorio.

\footnotetext{
* Autor de correspondencia:

Tel.: +51971623519

E-mail:miguelgallegos@upeu.edu.pe

DOI: https://doi.org/10.17162/riva.v7i1.1413

Recibido: 12/08/2021 Aceptado: 27/09/2020
} 
Palabras clave: Marketing relacional; confianza; comunicación; compromiso; manejo de conflictos

\section{Abstract}

The objective of this study is to determine the internal consistency and reliability of the Relationship Marketing instrument, by means of an exploratory analysis. The instrument, created by Ndubisi (2007), measures the dimensions of trust, commitment, communication, and conflict management using 16 items. Within the previous internal consistency analyses, a Cronbach's Alpha was obtained for the instrument, corresponding to $\square=0.883$ and 0.458 , $0.789,0.657,0.821$ for its dimensions. Thus, to verify the homogeneity of the variables, estimates were made in advance using $\mathrm{KMO}$, reaching an admissible Kaiser Meyer Olkin index of 0.816 and a Chi square of 695.946 with 120 degrees of freedom and significance level = $0.000(p<.001)$ by means of the Bartlett sphericity test, as an indispensable requirement for the extraction of factors by means of rotated components, acquiring loads for the first element between 0.780 and 0.790 ; for the second element, between 0.738 and 0.779 , for the third element, between 0.739 and 0.652 and for the fourth element 0.598 , justifying a high correlation, because they are greater than 0.4 , formalizing the objective of reviewing whether they are grouped empirically, as was predicted theoretically. However, items COMUN 10 (0.486), COMUN 11, (0.482), CONFL15 (0.148) did not obtain a load in their dimensions and items CONFI 5 (0.385), COMUN13 (0.244), CONFL16 (0.498) obtained additional loads in other dimensions, and thus could be eliminated, but they were kept in their theoretical factor, awaiting a confirmatory analysis.

Keywords: Relationship Marketing; trust; communication; commitment; conflict management.

\section{Introducción}

En ausencia de la práctica de técnicas y estrategias dirigidas a retener y optimizar la rentabilidad de clientes en las organizaciones. Es por ello que el marketing relacional sigue siendo un tema bastante abandonado por lo general en las empresas del Perú. Sin embargo, no es novedad que los problemas más frecuentes en las organizaciones sean por falta de solidez entre los empleados al momento de relacionarse con sus clientes (Barron,2011).

Por lo tanto, la variable desprende su valor, porque se despliega en el desarrollo de análisis, planificación, implementación, y monitoreo. El principal punto del marketing relacional es la ejecución y sustento de relaciones a largo plazo con los clientes, que se logran gracias a la creación de valores que para el cliente son muy beneficiosas y alcanzan su satisfacción (Ma \& Chen, 2008). Por otro lado, está basado en la interacción que se desarrollan entre las relaciones de vendedor-comprador, además sus valores centrales del marketing relacional 
son la colaboración y la creación de valor mutuo, incluyendo la observación de proveedores, clientes y otros como socios. (Gummesson, 2008). Ya que se ha descubierto que los clientes nuevos representan costos muy altos para la organización, es por ello que se da prioridad a la relación con los clientes antiguos.

El presente trabajo es un estudio que permite la inserción de la variable marketing relacional en el sector cine del Perú. Es por ello que Córdoba (2011) menciona que es necesario implementar en toda la estructura organizacional una perspectiva de marketing a largo plazo con el objetivo de saber las necesidades que el consumidor requiere y que valore las relaciones estables en los mercados. Así mismo para crear, brindar y mantener un proceso de transacciones beneficiosas con los compradores (Cutler, 2000, citado por Awill,Abdullahi, \& Abdulkadir, 2016).

Estudios realizados en el mundo sobre la variable marketing relacional se difunde que "la industria más estudiada es la de servicios financieros y seguros, con $20.38 \%$ del total; este porcentaje indica el claro sesgo de la investigación hacia el campo de los servicios. Le sigue el de comercio al por mayor, con 10. 19\%, las industrias manufactureras, con 5.73\%. Después se encuentran el comercio al por menor, las actividades recreativas, de servicio de comidas, salud humana y servicios administrativos, con $3.18 \%$ en general. Entre ellos Estados Unidos e Inglaterra con mayor participación en estudios aplicados entre 19.08\% y 18.32\%" (Wakabayashi, 2010).

Por otro lado, un estudio realizado en Perú señala que, dentro del presupuesto anual de las instituciones educativas privadas de Lima Metropolitana, el 88,9\% de los encuestados señalan que dentro de su presupuesto las actividades de marketing relacional representan menos del $5 \%$ del total. Específicamente de los distritos de San Juan de Miraflores, San Martín de Porres y Puente Piedra, concluyendo así que existe un impacto positivo del marketing relacional en los resultados organizacionales. (Salas Canales, 2017)

La población seleccionada para la validación del instrumento Relationship Marketing (RM) de Ndubisi (2007) en clientes de Cinemark S.A Surco, será de 100 usuarios frecuentes. Después de la inserción del instrumento los resultados serán el inicio para futuras investigaciones en el sector cine del Perú y así también generará un interés para las otras compañías ya que es breve y preciso con calidad internacional, reconocida. Además, los resultados captados indicarán la relación que tiene la empresa con sus clientes. La validación del instrumento se realizó mediante el análisis de coeficientes de correlación con el objeto de evaluar la construcción de las dimensiones y sus preguntas, además a través de un diseño no experimental, transversal. 
El estado de arte de la presente investigación comprende a diversos estudios de validación de la variable del marketing relacional, así como también si se realizaron estos estudios en Perú. Los presentes estudios acerca del instrumento del marketing relacional fueron realizados mediante validaciones y por lo general estás tienen un vínculo muy fuerte con la lealtad.

Estudios realizados en el sector bancario por Ndubisi (2007) en Malasia y por Husnain \& Akhtar, (2015) en Pakistan demostraron una validación de consistencia interna favorable del instrumento Relationship Marketing y sus dimensiones superando el coeficiente de 0.80 . Ademas existen otros estudios demostrando que el marketing relacional mediante sus dimensiones confianza, compromiso, comunicación y manejo de conflictos tienen un fuerte impacto relacionado con la lealtad (Siddig, 2015). En trabajos como de Sivesan (2012) la dimensión que mejor se relaciona con lealtad es la comunicación y dimensiones como compromiso y manejo de conflicto explican mejor la lealtad de los clientes (Awill, Abdullahi, \& Abdulkadir, 2016).

La variable marketing relacional se explica, en un conjunto de actividades de marketing orientados al establecimiento, acrecentamiento y mantenimiento de relaciones (Morgan y Hunt, 1994). El marketing relacional es una de las ramas muy importantes del marketing que, en la actualidad, se ha transformado en una estrategia eficaz e indispensable para que las organizaciones puedan mantener clientes satisfechos y con el impacto en la lealtad de sus clientes (Guzmán,2014). Sin embargo, Cordoba (2011) mencionan que el marketing relacional se manifiesta para destacar la necesidad de integrar en toda la organización una visión de marketing a largo plazo. Por lo tanto, el marketing relacional fue ganando lugar por la firme competencia en el entorno empresarial, que puede ser medido a través de las dimensiones de confianza, compromiso, comunicación y manejo de conflictos (Ndubisi,2007)

A nivel internacional se han aplicado y validado este instrumento en el sector de servicios (Ndubisi 2007; Husnain \& Akthar 2015; Siddig,et al. 2015; Sivessan, 2012). En Sudamérica no se encuentra ningún estudio sobre esta variable utilizando el RM. Además, en el Perú aún no existe la aplicación y validación del instrumento RM de Ndubisi (2007). Es por ello por lo que el presente trabajo propone la validación del instrumento Relationship Marketing en el sector cine del Perú, como en el caso de Cinemark de Surco de la ciudad de Lima.

El objetivo de esta investigación fue evaluar la consistencia y coherencia interna del instrumento Relationship Marketing "RM" de Ndubisi (2007) y sus dimensiones confianza, compromiso, comunicación, manejo de conflictos mediante coeficientes de correlación en los clientes de la empresa Cinemark de Surco de la ciudad de Lima. 


\section{Revisión de la literatura}

\subsection{Marketing relacional}

La variable marketing relacional del cliente han sido objeto de diversos estudios. En la actualidad existen intereses progresivos en el tema del marketing relacional. La firme competencia en el entorno empresarial ha dado lugar al levantamiento de relaciones más frecuentes y solidas entre la empresa y los clientes Ndubisi (2007).

Según Gordon (1998) citado por Engenharia, (2012), el marketing relacional es "el proceso consecutivo de encontrar y crear nuevos valores con clientes externos y compartir beneficios para ser disfrutados en la sociedad. El principal punto del marketing relacional es la ejecución y sustento de relaciones a largo plazo con los clientes, que se logran gracias a la creación de valores que para el cliente son muy beneficiosas y alcanzan su satisfacción, (Ma \& Chen,2008).

Gummesson, (2008) definió el marketing relacional como el marketing basado en la interacción entre las relaciones vendedor-comprador. Este autor sugiere que los valores centrales del marketing relacional son la colaboración y la creación de valor mutuo, incluida la observación de proveedores, clientes y otros como socios.

La gestión de marketing se puede definir como: "El análisis, planificación, implementación y monitoreo de programas para crear, brindar y mantener un proceso de transacciones beneficiosas con los compradores, con el fin de lograr las metas trazadas por la empresa. Cutler, (2000) citado por (Awill,Abdullahi, \& Abdulkadir, 2016).

Los conceptos de "marketing relacional" y "orientación al mercado" nacen para hacer hincapié en la importancia de implementar en toda la estructura organizacional una perspectiva de marketing a largo plazo que tenga en cuenta realmente las necesidades que el consumidor requiere y que valore la organización de relaciones estables en los mercados, (Cordoba 2011).

\subsection{Definición de dimensiones}

\subsubsection{Confianza.}

La confianza es descrita como, mediante el uso del sistema de seguridad en compras, así como las promesas relacionadas con el cliente. Morgan y Hunt (1994), la confianza es un elemento vital de las relaciones comerciales. Puede describirse como una fe o garantía con respecto a la intención de la segunda parte dentro del vínculo. (Lewicki, Roy \& McAllister, Daniel \& Bies, 1998). Importante por el desarrollo de relaciones generadoras de valor para ambas partes, (Renart 2001). En la perspectiva de Marketin relacional, la confianza se define, el aspecto de la relación comercial que establece el nivel en el que cada parte cree y puede 
confiar en la honestidad de la promesa ofrecida por la otra Callaghan et al., (1995) citado por (Saunders y Thornhill 2004).

\subsubsection{Compromiso.}

El compromiso se define en diversas formas, como a una aspiración a mantener una relación, Morgan y Hunt (1994), al comportamiento organizacional, (Meyer y Allen 1997). A esto se agrega que la dimensión conta de compromiso calculador y afectivo, (Chan y Ndubisi 2007; Grossman, 1998). El compromiso es otro determinante importante de la fuerza de la relación de marketing, y una construcción útil. Para medir la probabilidad de fidelidad del cliente y predecir la frecuencia de compra futura (Gundlach et al., 1995; Morgan y Hunt, 1994; Dwyer et al., 1987) citados por Dahie (2016). Webster, (1992) observó que el compromiso era el más común en diversos estudios del marketing relacional.

La literatura sobre marketing relacional está familiarizada con otro posible antecedente de lealtad del cliente, que es el compromiso de relación (Morgan y Hunt 1994); Bendapudi y Berry, 1997) citados por (Sivesan 2012).Basado en la literatura de comportamiento organizacional (Meyer y Allen 1997), el compromiso se define de diferentes maneras, como una aspiración a mantener una relación (Morgan y Hunt 1994) el abandono o el sacrificio si se rompe una relación (Anderson y Weitz, 1992), y con la falta de ofertas viables o atrayentes (Gundlach, Achrol y Mentzer, 1995) citado por (Husnain y Akhtar 2015) .Debido a estas variedades de base, se genera "adherencia" que mantiene a los clientes leales a la empresa.

\subsubsection{Comunicación.}

La comunicación se integra con las siguientes definiciones en diversos estudios realizados con respecto al de marketing relacional, interacciones web y otras interacciones relacionadas con información apropiada.

Ndubisi (2007), la comunicación es elemento esencial del marketing sea presencial o en las interacciones relacionadas. Como el ajuste de deseos y necesidades con la comunicación con los clientes. Schultz et al. (1992), citado por (Saunders \& Thornhill 2004). Morgan \& Hunt (1994) encontraron que la comunicación tiene un fuerte impacto en la confianza, y los describe como valores compartidos. Sosteniendo que la información apropiada a través de cartas, correo y otras interacciones generan valor en los clientes. Si estas comunicaciones son apropiadas, de apoyo, positivas, valiosas, simples y agradables, entonces se consideran "buenas".

\subsubsection{Manejo de conflictos.}

El manejo de conflictos se define con dos diferentes perspectivas, en primera instancia el conflicto puede ser destructiva o comunicacional. El manejo de conflictos es descrito como 
la capacidad de solución de problemas, donde se consigue la reducción de conflictos entre áreas de la empresa. Boonsathorn, (2007). El conflicto es un impacto negativo en la credibilidad y en la confianza de la empresa si el cliente lo ve como un manejo inapropiado y lento. (Ganesan, 1994) citado por (Auruskeviciene, Salciuviene, y Skudiene 2010). Por otro lado, el conflicto no es considerado como una experiencia dañina y destructiva por las personas, pero es un fenómeno más natural e invasivo en su comprensión, (Yau et al. 2000). El conflicto puede ser saludable y normal en una perspectiva organizacional. Putnam y Poole (1987) citado por (Abdullah 2013) consideran que el conflicto en el grado normal es desde un punto de vista comunicacional.

\subsection{Otros modelos de confiabilidad}

En este aspecto, se presentará el desarrollo de los diversos modelos, relationship marketing. En la investigación, el instrumento Relationship Marketing, fue desarrollado en primer lugar por Ndubisi (2007), en la universidad de Monash en Malasia, construida con cuatro dimensiones, compromiso, comunicación, confianza y manejo de conflictos, con una escala de Likert de cinco puntos. Además, el instrumento Relationship Marketing, tiene un impacto, que crea, refuerza y retiene mediante planes de marketing dirigidos a la lealtad de los clientes. Ndubisi (2007) registro su investigación en la universidad de malasia en el idioma inglés, el instrumento de su investigación fue muy usado en Sri Lanka, Pakistan, EE.UU, resultando efectos positivos en los países mencionados.

Actualmente no se encuentra adaptación al español del estudio RM de Ndubisi (2007), ya que en Sudamérica no hay estudios utilizados de la validación del instrumento RM de Ndubisi (2007). Es por ello por lo que el presente trabajo propuso la validación del instrumento Relationship Marketing en el sector cine del Perú, como en el caso de Cinemark de Surco de la ciudad de Lima.

\section{Materiales y Métodos}

\subsection{Diseño}

El tipo estudio es correlacional de diseño no experimental porque se buscó establecer relación entre la variable marketing relacional y lealtad en los clientes de Cinemark S.A de Surco. Ya que el diseño es una estrategia con tácticas que se desarrolla en la recolección de información que se requiere en la investigación, y este debe ser flexible y adaptable a los cambios que pueden presentarse durante el desarrollo de esta investigación (Hernández, Fernández, y Baptista, 2010). 


\subsection{Participantes}

La población seleccionada para fines de la validación del instrumento "RM" será de 100 clientes de la empresa Cinemark S.A de Surco de la ciudad de Lima, considerándose como una muestra por conveniencia. En este aspecto depende de características de la presente investigación y se infiere un procedimiento de selección informal pues no será necesario el uso den fórmulas de probabilidad, sino del proceso de toma de decisiones". Fernandez y Baptista (2010).

\subsection{Instrumentos}

La técnica para la recolección de datos será utilizada mediante encuestas digitales. Se gestionará una carta formal al área comercial de atención al cliente de la empresa Cinemark S.A de Surco solicitando el permiso formal de aplicar la encuesta a los clientes frecuentes de Cinemark S.A de Surco de la ciudad de Lima, durante una semana aprovechando la libre afluencia de clientes en el local de empresa. Al abordar a los clientes, se saluda proactivamente, solicitando un poco de su tiempo, para que pueda llenar la encuesta que será a través de una Tablet.

Para la medición de la variable "el perfil del marketing relacional", se utilizó el instrumento de escala "RM", creado por Ndubisi (2007). El presente cuestionario consta de 4 dimensiones, confianza (5 ítems), compromiso (3 ítems), comunicación (4 ítems) y manejo de conflictos. Presenta una escala de Likert de 5 puntos; Completamente de acuerdo $=5$, De acuerdo $=4$, Indiferente $=3$, Desacuerdo $=2$ y Completamente desacuerdo $=1$.

Este cuestionario fue utilizado por Husnain y Akhtar (2015) en una muestra de 220 clientes bancarios de Pakistan con una prueba de confiabilidad de 0.89. A su vez el instrumento "RM" fue utilizado por Sivesan (2012) aplicado a 102 clientes bancarios de la universidad de Jaffna de la ciudad de Sri. Lanka.con una prueba de confiabilidad de 0.76 .

\subsection{Procedimiento y análisis de datos}

Se tabulará la información de la encuesta RM directamente en Excel para luego importarlo a la plantilla del software con el que trabajaremos, se editó las etiquetas de las dimensiones por códigos de reconocimiento para facilitar el análisis y la obtención de los resultados. Para el procesamiento de datos se utilizará el Software Estadístico IBM SPSS y para el análisis de la confiabilidad se utilizará el coeficiente de Alpha de Cronbach y análisis factorial a fin de evaluar las consistencias internas del total del instrumento, dimensiones y de cada pregunta (Oviedo \& Campos-Arias, 2005). 


\section{Resultados}

A continuación, se presentan los resultados de la investigación. Se caracteriza a los participantes a partir de algunas variables demográficas. Para la validación del instrumento, en la primera fase se presentan resultados del análisis de consistencia interna mediante el Alpha de Cronbach. En la segunda fase se realiza el análisis factorial con resultados previos de estimaciones mediante KMO y esfericidad de Barlett, para obtener los resultados de componentes rotados y así evaluar la homogeneidad de los reactivos respecto a sus componentes o factores.

\subsection{Resultados demográficos}

En la tabla 1 se muestran las características demográficas, de los clientes de Cinemark, la mayoría de los clientes es de género femenino (62\%). El 55\% de los clientes tiene un rango en edad de 24 a 29 años, a esto le sigue el $44 \%$ de los clientes de 18 a 23 años, de 30 a 35 no tienen efecto, y de 36 años a más solo representa al 1\%. Además, podemos ver que el sexo femenino representa el $62 \%$ de los clientes frecuentes frente al $38 \%$ del sexo masculino. Así mismo podemos observar que el tiempo sobresaliente de relación con Cinemark es de 1 a 3 años con un $65 \%$.

\section{Tabla 1}

Características demográficas

\begin{tabular}{llcc}
\hline & & Recuento & $\begin{array}{c}\% \text { de N } \\
\text { columnas }\end{array}$ \\
\hline \multirow{2}{*}{ Género } & Masculino & 38 & $38 \%$ \\
& Femenino & 62 & $62 \%$ \\
& 18 a 23 años & 44 & $44 \%$ \\
Edad & 24 a 29 años & 55 & $55 \%$ \\
Es usted afiliado o cliente & 30 a 35 años & 0 & $0 \%$ \\
frecuente de Cinemark & 36 años a mas & 1 & $1 \%$ \\
Símpo de relación con & No & 100 & $1 \%$ \\
Cinemark & Menos de un año & 0 & $0 \%$ \\
& 1 a 3 años & 15 & $15 \%$ \\
& 4 a 6 años & 65 & $65 \%$ \\
& 7 a 9 años & 16 & $16 \%$ \\
\hline
\end{tabular}


$1 \%$

\subsection{Análisis del Alpha de Cronbach}

En la tabla 2 se presenta la prueba de confiabilidad del instrumento RM con el objetivo de medir la variable relationship marketing a través de la prueba piloto. Mediante el Alpha Cronbach con un coeficiente de 0.883 , demostrando una consistencia aceptable para la recolección de datos. Además, se presenta la prueba de confiabilidad de las dimensiones del instrumento que mide la variable Marketing Relacional a través de la prueba piloto. Mediante el Alpha de Cronbach se obtuvo los siguientes coeficientes: confianza (0.458), compromiso (0.789), comunicación (0.657) y manejo de conflictos (0.821). Las dimensiones manejo de conflictos y compromiso aparecen con una mayor correlación.

\section{Tabla 2}

Prueba de confiabilidad del instrumento de Marketing relacional y dimensiones

\begin{tabular}{lcc}
\hline & Alfa de Cronbach & N de elementos \\
\hline Confianza & .458 & 5 \\
Compromiso & .789 & 4 \\
Comunicación & .657 & 4 \\
Manejo de conflictos & .821 & 3 \\
Marketing relacional & .883 & 16 \\
\hline
\end{tabular}

En la Tabla 3 se muestra la prueba de confiabilidad de cada uno de los ítems del instrumento que mide la variable marketing relacional de los clientes de Cinemark. Todos los ítems obtuvieron un valor mayor al 0.3 demostrando confiabilidad de las preguntas en la recopilación de datos, fluctuando entre 0.870 y 0.884 . Por lo tanto, se infiere que las preguntas son los ingredientes importantes para el desarrollo de la presente investigación, que busca relacionarse con los clientes a largo plazo.

\section{Tabla 3}

Prueba de confiabilidad de los ítems del instrumento que mide la variable Marketing relacional

\begin{tabular}{ccccc}
\hline & \multicolumn{2}{c}{$\begin{array}{c}\text { Media de escala Varianza de escala } \\
\text { si el elemento se } \\
\text { ha suprimido }\end{array}$} & $\begin{array}{c}\text { Correlación } \\
\text { total de } \\
\text { ha suprimido } \\
\text { elementos } \\
\text { corregida }\end{array}$ & $\begin{array}{c}\text { Alfa de Cronbach si } \\
\text { el elemento se ha } \\
\text { suprimido }\end{array}$ \\
\hline Item 1 & 58,77 & 36,157 &, 504 &, 877 \\
Item2 & 58,68 & 37,484 &, 303 &, 884 \\
Item3 & 58,39 & 40,176 &,- 087 &, 897 \\
Item4 & 58,64 & 35,034 &, 525 &, 876
\end{tabular}




\begin{tabular}{lllll} 
Item5 & 58,59 & 34,560 &, 615 &, 872 \\
Item6 & 58,76 & 32,731 &, 680 &, 869 \\
Item7 & 58,78 & 34,341 &, 615 &, 872 \\
Item8 & 58,72 & 35,215 &, 579 &, 874 \\
Item9 & 58,83 & 34,309 &, 600 &, 873 \\
Item10 & 58,51 & 35,747 &, 458 &, 879 \\
Item11 & 58,43 & 37,405 &, 349 &, 882 \\
Item12 & 58,61 & 33,187 &, 698 &, 868 \\
Item13 & 58,67 & 35,593 &, 522 &, 876 \\
Item14 & 58,63 & 34,089 &, 672 &, 870 \\
Item15 & 58,74 & 35,479 &, 633 &, 873 \\
Item16 & 58,70 & 33,539 &, 732 &, 867 \\
\hline
\end{tabular}

\subsection{Análisis factorial}

En tabla 4 se puede apreciar los reactivos según ítems y factores por dimensión. Los presentes reactivos o preguntas del instrumento se encuentran codificadas, CONFI para la dimensión confianza, COMP para la dimensión compromiso, COMU para la dimensión comunicación y MC para la dimensión manejo de conflictos.

\section{Tabla 4}

Distribución de ítems por factores

\begin{tabular}{|c|c|c|}
\hline Ítems & Reactivos & Factores \\
\hline 1 & $\begin{array}{l}\text { Cinemark está muy preocupado con la seguridad de mis } \\
\text { compras. }\end{array}$ & Confianza (CONFI) \\
\hline 2 & $\begin{array}{l}\text { Las promesas de Cinemark son confiables y es consciente } \\
\text { en brindar un servicio de calidad. }\end{array}$ & Confianza (CONFI) \\
\hline 3 & Los empleados de Cinemark muestran respeto a los clientes & Confianza (CONFI) \\
\hline 4 & $\begin{array}{l}\text { Cinemark cumple con sus responsabilidades con los } \\
\text { clientes. }\end{array}$ & Confianza (CONFI) \\
\hline 5 & Tengo confianza con el servicio de Cinemark. & Confianza (CONFI) \\
\hline 6 & Cinemark se adapta para satisfacer mis necesidades. & Compromiso (COMP) \\
\hline 7 & $\begin{array}{l}\text { Cinemark ofrece servicios personalizados para satisfacer } \\
\text { mis necesidades. }\end{array}$ & Compromiso (COMP) \\
\hline 8 & $\begin{array}{l}\text { Cinemark es adaptable cuando se cambian sus productos y } \\
\text { servicios. }\end{array}$ & Compromiso (COMP) \\
\hline 9 & Cinemark es flexible para atender mis necesidades. & Compromiso (COMP) \\
\hline 10 & La publicidad de Cinemark proporciona información & Comunicación \\
\hline
\end{tabular}


oportuna y confiable

11 Cinemark proporciona información cuando hay un nuevo servicio.

12 Cinemark hace y cumple promesas.

13 La información proporcionada por Cinemark es precisa.

14 Cinemark intenta evitar posibles conflictos.

15 Cinemark intenta resolver conflictos manifiestos antes de que generen problemas.

16 Cinemark tiene la capacidad de discutir abiertamente las soluciones cuando los problemas surgen.
(COMU)

Comunicación

(COMU)

Comunicación

(COMU)

Comunicación

(COMU)

Manejo de conflictos

$(\mathrm{MC})$

Manejo de conflictos

$(\mathrm{MC})$

Manejo de conflictos

$(\mathrm{MC})$

En la tabla 5 se muestra la medida de adecuación muestral KMO obteniendo un valor superior a 0.6 (<0.816), lo que apunta que la correlación entre los ítems tiene la mayor fuerza para la extracción de los factores, a su vez señala al instrumento marketing relacional. Así mismo, mediante la prueba de esfericidad de Bartlett (695.946), se demuestra la consistencia interna del instrumento marketing relacional ya que es estadísticamente significativo, pues el nivel de significancia es menor a 0.005 (>0.000) indicando de esa manera que las correlaciones no son iguales a 0 .

\section{Tabla 5}

Prueba de KMO y Barlett

Medida Kaiser-Meyer-Olkin de adecuación de muestreo

Prueba de esfericidad de Bartlett

gl

Sig.

.000

En la Tabla 6 se puede observar la agrupación de las preguntas con sus respectivas dimensiones o componentes. El resultante señala que existen 4 componentes, que coinciden con las dimensiones que tiene el instrumento. Por lo tanto, para el componente confianza, las preguntas que cargan son: CONFI1, CONFI2, CONFI3, CONFI4, CONFI5; seguido el componente compromiso, las preguntas que cargan son COMPR6, COMPR7, COMPR8, COMUN10, COMUN11, COMUN11, para el componente comunicación, COMUN12, COMUN13, COMFL14, y para el componente manejo de conflictos, CONFL16. De esta manera se debe considerar que CONFI5 (0.385), toma carga adicional en el segundo 
componente; el COMUN13, en el primer componente y CONFL16, en el primer componente, además, COMUN 10, COMUN 11 yCONFL15, no están tomando valores en su respectiva dimensionalidad, pero obtiene valores en el segundo y primer componente.

\section{Tabla 6}

Matriz de componentes rotados

\begin{tabular}{ccccc}
\hline \multicolumn{5}{c}{ Componente } \\
\hline No & Confianza & Compromiso & Comunicación & $\begin{array}{l}\text { Manejo de } \\
\text { Conflictos }\end{array}$ \\
\hline CONFI1 & .790 & & \\
CONFI2 & .780 & & \\
CONFI3 & .723 & & \\
CONFI4 & .667 & & \\
CONFI5 & .448 & .385 & \\
COMPR6 & & .779 & \\
COMPR7 & & .758 & \\
COMPR8 & & .738 & \\
COMPR9 & & .699 & & \\
COMUN10 & & .486 & .739 & \\
COMUN11 & & .482 & .592 \\
COMUN12 & & & \\
COMUN13 & .244 & & \\
CONFL14 & & & \\
CONFL15 & .148 & & \\
CONFL16 & .498 & & \\
\hline
\end{tabular}

Método de extracción: análisis de componentes principales. Método de rotación: Varimax con normalización Kaiser. a. La rotación ha convergido en 6 iteraciones.

\section{Discusión}

El propósito de la presente investigación fue evaluar la validez del instrumento Relationship Marketing, encontrándose que tiene consistencia interna y que evidencia confiabilidad para asegurar que los datos que se recogen con el instrumento RM sean confiables.

Para la consistencia interna del instrumento en su totalidad (16 reactivos) se obtuvo un Alpha de Cronbach de 0.88 en 100 clientes de Cinemark, Surco de la ciudad de lima. En 
la investigación de Husnain y Aktar (2015), se aplicó el mismo instrumento a los clientes bancarios de Pakistan obteniendo un Alpha de Cronbach de 0.89 mayor al coeficiente 0.883 de la presente investigación. Además, también en la investigación de (Awill, Abdullahi, \& Abdulkadir, 2016), aplicado a 100 clientes de Beco Powering Somalia en Mogadisco-Somalia con un Alpha de Cronbach de 0.80. Por También Siddig, A. (2015) en su estudio a 238 clientes bancarios en el estado de Jartum, Sudán con un Alpha de Cronbach de 0.80. Por Sivessan (2012), con un Alfa de Cronbach 0.753 aplicado a 102 clientes bancarios en Jaffna.

En la Tabla 2 se presenta la prueba de confiabilidad de las dimensiones del instrumento que mide la variable Marketing Relacional a través de la prueba piloto. Mediante el Alpha de Cronbach se obtuvo los siguientes coeficientes: confianza (0.458), compromiso (0.789), comunicación (0.657) y manejo de conflictos (0.821). Las dimensiones manejo de conflictos y compromiso aparecen con una mayor correlación. En comparación con una investigación aplicada en Malasia por Ndubisi (2007), con el presente instrumento obtuvieron según su análisis los siguientes coeficientes: confianza (0.458), compromiso (0.789), comunicación (0.657) y manejo de conflictos $(0.821)$.

Por lo tanto, el signo positivo de las estimaciones muestra que mientras más alto sea el alcance de estos fundamentos, mayor será la fuerza de relación con los clientes, es por ello por lo que todos los elementos de la investigación mencionada son firmemente respaldados.

En la dimensión confianza (tabla 2) se obtuvo un $\alpha=0.58$, en la investigación de Ndubisi (2007) con un $\alpha=0.84$. Por otro lado, estudios como el de Husnain \& Akhtar, (2015) con un $\alpha=0.80$, así como el de Siddig, A. (2015) declaran en su investigación un $\alpha=0.65$, y en la investigación de Abdulla \& Kanyan (2013) con un $\alpha=0.82$. y por último en el estudio de Sivanandamoorthy y Achchuthan (2013) con un $\alpha=0.76$, concluyendo así que el instrumento puede medir la confianza la confianza que es descrita como, mediante el uso del sistema de seguridad en compras, así como las promesas relacionadas con el cliente. Morgan y Hunt (1994), la confianza es un elemento vital de las relaciones comerciales. Puede describirse como una fe o garantía con respecto a la intención de la segunda parte dentro del vínculo. (Lewicki, Roy \& McAllister, Daniel \& Bies, 1998).

Para la dimensión compromiso (Tabla 2) se obtuvo un $\alpha=0.78$, en el estudio de Ndubisi (2007) con un $\alpha=0.84$, sin embargo en la investigación de Siddig, A. (2015) se obtuvo un $\alpha=0.64$; seguido la investigación de Husnain \& Akhtar, (2015) se obtuvo un $\alpha=0.58$, en el estudio de Abdulla \& Kanyan (2013) con un $\alpha=0.84$, y en la investigación de Sivanandamoorthy y Achchuthan (2013) con un $\alpha=0.78$, Se demuestra así que, el instrumento puede medir el compromiso ya que este se define en diversas formas: como a una aspiración a mantener una relación, (Morgan y Hunt, 1994). 
Por otro lado, para la dimensión de comunicación (Tabla 2) se obtuvo un $\alpha=0.65$.En el estudio de Ndubisi (2007) se obtuvo $\alpha=0.78$. A esto se compara la investigación de Siddig, A. (2015) con un $\alpha=0.656$., seguido de Husnain \& Akhtar, (2015) que obtuvo un $\alpha=0.80$, y con mayor presencia, se encuentra la investigación de Abdulla \& Kanyan (2013) , con un $\alpha=0.90$ ademas la investigación de Sivanandamoorthy y Achchuthan (2013) con un $\alpha=0.72$, de tal manera que se puede medir la comunicación por los mismo que es un elemento esencial del marketing sea presencial o en las interacciones relacionadas. (Ndubisi,2007) Como el ajuste de deseos y necesidades con la comunicación con los clientes. Schultz et al. (1992), citado por (Saunders \& Thornhill 2004).

Por último, en la dimensión manejo de conflictos (Tabla 2) se obtuvo un $\alpha=0.82$, en el estudio de Ndubisi (2007) con un $\alpha=0.73$, sin embargo, en el estudio de Siddig, A. (2015) obtuvo un $\alpha=0.49$, seguido Abdulla \& Kanyan (2013) se obtuvo un $\alpha=0.49$, en la investigación de Husnain \& Akhtar, (2015) obtienen un $\alpha=0.621$. y en la investigación de Sivanandamoorthy y Achchuthan (2013) alcanzo un $\alpha=0.77$, es por ello por lo que la dimensión conflicto se puede mediar ya que es descrito como la capacidad de solución de problemas, donde se consigue la reducción de conflictos entre áreas de la empresa. Boonsathorn, (2007). Además, es un impacto negativo en la credibilidad y en la confianza de la empresa si el cliente lo ve como un manejo inapropiado y lento. (Ganesan, 1994) citado por (Auruskeviciene, Salciuviene, y Skudiene 2010).

Posteriormente al análisis de exploratorio se realizó la prueba de esfericidad y de correlaciones para su extracción de factores que agrupen sus respectivos reactivos según los componentes que corresponda. Para el análisis del instrumento se ha propuesto 4 dimensiones, según el modelo de Ndubisi (2007), obteniéndose un índice de Kaiser Meyer Olkin (KMO) aceptable de 0.816 y un Chi cuadrado de 695.946 con 120 grados de libertad y nivel de significancia $=0.000(p<001)$ mediante la prueba de esfericidad de Barlett. Las estimaciones mencionadas aseguran que las correlaciones entre una o más variables (ítems o reactivos) son adecuados, como requisito de la ejecución del análisis factorial Cortina, (1993). Por lo tanto, la importancia y el significado del instrumento no siempre se podrá observar la homogeneidad que implica, que los reactivos, se agrupan a los factores teóricos, (Clarke y Belk 1978).

Si bien es cierto las correlaciones entre los ítems demuestran la consistencia interna del instrumento, no evidencia la homogeneidad que consiste en que los reactivos o variables revisen o evalúen estrictamente el componente (Cortina ,1993), en ese sentido ha sido necesario realizar el análisis factorial mediante componentes rotados para verificar si los reactivos se agrupan a los factores teóricos (Clark \& Watson, 1995). Por lo tanto, las dimensionalidades o comunalidades reciben cargas agrupan a los factores teóricos las 
dimensionalidades o comunalidades (dimensiones) han recibido cargas que se agrupan con valores cercanos.

Para el primer elemento se reciben valores entre 0.780 y 0.790 , para el segundo elemento, valores entre 0.758 y 0.779 , para el tercer elemento valores entre 0.739 y 0.652 para el cuarto elemento, valores de 0.598 , demostrando evidencia de alta correlación, porque son mayores a 0.4 , eliminando cargas menores a 0.3-0.25, cumpliendo el objetivo de ver si se agrupan empíricamente, tal como, teóricamente se había pronosticado (Nunnally \& Berstein 1995).Sin embargo, el reactivo COMUN 10 (0.486), COMUN 11, (0.482), CONFL15 (0.148) no obtuvieron carga en su dimensionalidad y los reactivos CONFI 5 (0.385), COMUN13 (0.244), CONFL16 (0.498) obtuvieron cargas adicionales en otros elementos, pudiendo ser eliminados, se decidió mantenerlos en su factor teórico, esperando un análisis confirmatorio.

La consistencia interna y la homogeneidad demuestra que el instrumento de Ndubisi (2007) puede obtener datos empíricamente de acuerdo con los factores teóricos propuestos del marketing relacional que implica la percepción del trabajador respecto al valor intrínseco que le da a su trabajo.

\section{Conclusiones}

En conclusión, la investigación actual, define que mediante los argumentos teóricos y resultados de la variable marketing relacional de Ndubisi (2007) se da la consistencia de las evidencias requeridas para la medición del constructo del marketing relacional en la empresa Cinemark de surco, así también en otras organizaciones.

\section{Agradecimientos}

Este apartado llega gracias a la participación y colaboración de los profesores y compañeros de la universidad peruana unión para llegar a cumplir con el objeto de la presente investigación.

\section{Referencias}

Abdullah, F. (2013). Managing the dimensions of relationship marketing in the food service industry. Jurnal pengurusan 37, 91-103.

Auruskeviciene, Vilte, Laura Salciuviene, y Vida Skudiene,(2010). The relationship quality effect on customer loyalty. ISM University of management and economics 10, 23. https://doi.org/10.18002/pec.v0i10.637 
Awill,K. Abdullahi,M. \& Abdulkadir,D. (2016). Relationship between marketing and customer satiscfaction. Multidisciplinary reseacrh 2, 2-10.

Barron. 2011. Marketing relacional como estrategia. Quipukamayoc de la unmsm 19, 57-62.

Clarke, Thomas, y Russell Belk. (1978). The effects of product involvement and task definition on anticipated consumer effort. Faculty Working Papers.

Cordoba, J. 2011. Del marketing transaccional al marketing relacional. Transactional marketing to relationship marketing 5(1), 13-26. https://doi.org/10.4272/978-84-9745184-0.ch1.

Cortina, Jose M. (1993). What is coefficient alpha? An examination of theory and applications 78(1), 98-104.

Feiteira, Bruno. (2012). Marketing relacional numa empresa de serviços. Tesis de maestria. Universidade do Minho.

Gummesson, Evert. (2008). New Economy Relationship Marketing in the New Economy, October 2012, 37-41.

Guzmán, J. (2014). Estrategia de marketing relacional para lograr la fidelización de los clientes. Relational marketing strategy to achieve customer loyalty $0(2), 25-42$. https://doi.org/10.17162/au.v0i2.71.

Hernández, Roberto, Carlos Fernández, y Pilar Baptista. (2010). Metologia de la investigación. Editado por Hill Mc Graw. Quinta edi. México.

Hugo, Salas. (2017). Influencia del marketing relacional en los resultados organizacionales de instituciones educativas privadas de Lima metropolitana (Perú). Revista de investigaciones altoandinas - journal of high andean research 19(1), 63-74. https://doi.org/10.18271/ria.2017.256.

Husnain, Mudassir, y Waheed Akhtar. (2015). Relationship marketing and customer Loyalty: evidence from banking sector in pakistan. Global journal of management and business research 15(10), 1-14. https://doi.org/10.1016/j.urology.2004.06.042.

Lewicki, Roy \& McAllister, Daniel \& Bies, Robert. (1998). Trust and distrust: new relationships and realities». Mapbox 23(3), 438-58. https://doi.org/10.2307/259288.

Ma, Ming, y Jinyuan Chen. (2008). Phase-type distribution of customer relationship with markovian response and marketing expenditure decision on the customer lifetime value. Marketing relationship 187: 313-26. https://doi.org/10.1016/j.ejor.2007.03.018.

Meyer, P. \& Allen, J. (1997). Commitment in the workplace: theory. Research and application. Thousand Oaks, CA: SAGE Publications, Inc. http://dx.doi.org/10.4135/9781452231556

Morgan, R. y, y S. Hunt. (1994). The commitment-trust theory of relationship marketing. Journal of marketing 58(3), 20-38. https://doi.org/10.2307/1252308.

Ndubisi, Nelson Oly. (2007). Relationship marketing and customer loyalty. Marketing Intelligence and Planning 25(1), 98-106. https://doi.org/10.1108/02634500710722425. 
Nunnally \& Berstein. (1995). Teoría Psicométrica. México: McGraw Hill.

Oviedo, H. C., \& Campo-Arias, A. (2005). Aproximación al uso del coeficiente alfa de cronbach. $\begin{array}{llll}\text { Revista colombiana de psiquiatría 34(4), } & \text { 572-80. }\end{array}$ http://www.redalyc.org/pdf/806/80634409.pdf

Renart, L. (2001). Marketing relacional: oportunidades en internet. Revista de antiguos alumnos 31, 30-35. http://www.ee-iese.com/82/82pdf/afondo3.pdf.

Saunders, Mark, y Adrian Thornhill. (2004). Trust and mistrust in organizations: an exploration using an organizational justice framework. European journal of work and organizational psychology 13(4), 493-515. https://doi.org/10.1080/13594320444000182.

Siddig, A., et al. (2015). Customer relationship management quality and customer loyalty: evidence from sudanese bank customers. Academic research international 6(1): 259-69. www.savap.org.pk\%0Awww.journals.savap.org.pk.

Sivanandamoorthy, Sivesan, y Sivapalan Achchuthan. (2013). Research paper customer perception towards relationship marketing and personal demographic variables: a study of jaffna district, srilanka, October 2013.

Sivesan, S. (2012). Impact of Relationship Marketing on Customers loyalty on banking sectors. South asian academic research journals 2(3), 179-91. Wakabayashi, José Luis. (2010). La investigación sobre el marketing relacional: un análisis de contenido de la literatura 2007-2008. Journal of Economics, Finance and Administrative Science 15(29), 119-30.

Webster (1992) The Changing Role of Marketing in the Corporation.pdf». Marketing Relationship. https://journals.sagepub.com/doi/10.1177/002224299205600402.

Yau, Oliver H.M., Peter R. McFetridge, Raymond P.M. Chow, Jenny S.Y. Lee, Leo Y.M. Sin, y Alan C.B. Tse. 2000. Is relationship marketing for everyone? European Journal of Marketing 34(9/10), 1111-27. https://doi.org/10.1108/03090560010342494. 